PACS: $05.70 . \mathrm{Ce}$

\title{
DETERMINATION OF THERMODYNAMIC QUANTITIES OF BINARY METAL SYSTEMS WITH LOW SOLUBILITY OF COMPONENTS IN THE SOLID $\alpha$-PHASE
}

\author{
A.P. Shcherban, O.A. Datsenko \\ National Scientific Center "Kharkov Institute of Physics and Technology" \\ 1, Academicheskaya st., Kharkov, 61108, Ukraine \\ E-mail: shcherban@kipt.kharkov.ua, https://orcid.org/0000-0002-6004-0579 \\ Received June 7, 2018; accepted September 10, 2018
}

The method for determining the thermodynamic quantities: the partial enthalpy of dissolution $\Delta \bar{H}_{B}^{\alpha}$, the activity coefficients $\gamma_{B}^{\alpha}$ and activities $a_{B}^{\alpha}$ of the second component B in binary metallic systems with low solubility in the solid $\alpha$-phase has been proposed. The algorithm for determining the above values is a sequence of preliminary calculations of the limiting and equilibrium distribution coefficients and construction of saturated solid solution lines for such systems with low solubility of the second component. Preliminarily obtained data are necessary for determining the partial enthalpy of dissolution and other quantities in the systems under study. Such a sequence of calculations is the proposed method for determining the thermodynamic parameters of dissolution. The values and temperature dependences of the dissolution enthalpy of component having a low solubility for such systems as Cd-Na, $\mathrm{Cd}-\mathrm{Tl}, \mathrm{Te}-\mathrm{Ga}, \mathrm{Te}-\mathrm{As}, \mathrm{Te}-\mathrm{Cu}, \mathrm{Zn}-\mathrm{Sn}$, as well as the activity coefficients $\gamma_{B}^{\alpha}$ and the activities $a_{B}^{\alpha}$ of the second component in the saturating $\alpha$-phase in these systems were determined. An analysis of obtained results on the enthalpy of dissolution shows that a change in the partial enthalpy with a change in the dissolution temperature is observed. When the temperature decreases from the melting point of the pure main component to the eutectic temperature, an increase in the partial enthalpies of dissolution of the second component is observed for studied systems. The Te-Ga system is characterized by a negative value $\Delta \bar{H}_{B}^{\alpha}$. A negative value $\Delta \bar{H}_{B}^{\alpha}$ indicates an exothermic process of gallium dissolution in tellurium, in contrast to other systems in which the dissolution of the second component occurs with heat absorption. Analyzing the enthalpy of dissolution in the systems under study, it should be noted that the low values of $\Delta \bar{H}_{B}^{\alpha}<2000 \mathrm{~J} / \mathrm{mol}$ are characterized by the systems Cd-Na and Te-Ga. For systems Te-Cu, Te-As, TeTl, Zn-Sn $\Delta \bar{H}_{B}^{\alpha}>15000 \mathrm{~J} / \mathrm{mol}$. Systems with a value $\Delta \bar{H}_{B}^{\alpha}>15000 \mathrm{~J} / \mathrm{mol}$ reveal a tendency to the occurrence of retrograde solubility in them. The difference in values $\gamma_{B}^{\alpha}$ is found to be more than two orders of magnitude for different systems.

KEY WORDS: binary metal systems, $\alpha$-phase, dissolution enthalpy, activity coefficient, activity, retrograde solubility, phase diagram

\section{ОПРЕДЕЛЕНИЕ ТЕРМОДИНАМИЧЕСКИХ ВЕЛИЧИН БИНАРНЫХ МЕТАЛЛИЧЕСКИХ СИСТЕМ С НИЗКОЙ

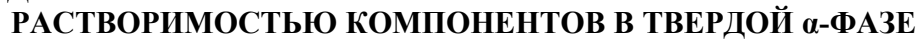

А.П. Щербань, О.А. Даценко

Национальный Научный Центр “Харьковский физико-технический институт ул. Академическая 1, г. Харьков, 61108, Украина

Предложен метод определения термодинамических величин: парциальной энтальпии растворения $\Delta \bar{H}_{B}^{\alpha}$, коэффициентов активности $\gamma_{B}^{\alpha}$ и активностей $a_{B}^{\alpha}$ второго компонента В в двойных металлических системах с низкой растворимостью в твердой $\alpha$-фазе. Алгоритм определения выше указанных величин заключается в последовательности предварительных расчетов предельных и равновесных коэффициентов распределения и построения линий насыщенного твердого раствора для таких систем с низкой растворимостью второго компонента. Предварительно полученные данные являются необходимыми для определения парциальной энтальпии растворения $\Delta \bar{H}_{B}^{\alpha}$ и других величин в исследуемых системах. Такая последовательность расчетов является предлагаемым методом определения термодинамических параметров растворения. Определены значения и температурные зависимости энтальпии растворения малорастворимого компонента для таких систем как Cd$\mathrm{Na}, \mathrm{Cd}-\mathrm{Tl}, \mathrm{Te}-\mathrm{Ga}, \mathrm{Te}-\mathrm{As}, \mathrm{Te}-\mathrm{Cu}, \mathrm{Zn}-\mathrm{Sn}$, а также коэффициенты активности $\gamma_{B}^{\alpha}$ и активности $a_{B}^{\alpha}$ второго компонента в насыщенной $\alpha$-фазе в этих системах. Анализ полученных результатов по энтальпии растворения показывает, что наблюдается изменение парциальной энтальпии с изменением температуры растворения. При снижении температуры от точки плавления чистого основного компонента до температуры эвтектики наблюдается увеличение парциальных энтальпий растворения второго компонента для исследованных систем. Система Те-Ga характеризуется отрицательным значением $\Delta \bar{H}_{B}^{\alpha}$. Отрицательное значение $\Delta \bar{H}_{B}^{\alpha}$ указывает на экзотермический процесс растворения галлия в теллуре, в отличие от других систем, в которых растворение второго компонента происходит с поглощением тепла. Анализируя значения энтальпии растворения в исследуемых системах, необходимо отметить, что низким значением $\Delta \bar{H}_{B}^{\alpha}<2000$ Дж/моль характеризуются системы СdNa и Te-Ga. Для систем Te-Cu, Te-As, Te-Tl, Zn-Sn $\Delta \bar{H}_{B}^{\alpha}>15000$ Дж/моль. Системы со значением $\Delta \bar{H}_{B}^{\alpha}>15000$ Дж/моль выявляют тенденцию к возникновению в них ретроградной растворимости. Выявлено различие в значениях $\gamma_{B}^{\alpha}$ более, чем на два порядка для различных систем. 
КЛЮЧЕВЫЕ СЛОВА: двойные металлические системы, $\alpha$-фаза, энтальпия растворения, коэффициент активности, активность, ретроградная растворимость, диаграмма состояния

\section{ВИЗНАЧЕННЯ ТЕРМОДИНАМІЧНИХ ВЕЛИЧИН БІНАРНИХ МЕТАЛЕВИХ СИСТЕМ 3 НИЗЬКОЮ

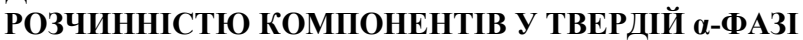 \\ О.П. Щербань, О.А. Даценко \\ Наиіональний Науковий Центр “Харківський фізико-технічний інститут” вул. Академічна 1, м. Харків, 61108, Украӥна}

Запропоновано метод визначення термодинамічних величин: парціальної ентальпії розчинення $\Delta \bar{H}_{B}^{\alpha}$, коефіцієнтів активності $\gamma_{B}^{\alpha}$ та активностей $a_{B}^{\alpha}$ другого компонента В в подвійних металевих системах з низькою розчинністю у твердій $\alpha$-фазі. Алгоритм визначення вище зазначених величин полягає в послідовності попередніх розрахунків граничних і рівноважних коефіцієнтів розподілу і побудови ліній насиченого твердого розчину для таких систем з низькою розчинністю другого компонента. Попередньо отримані дані є необхідними для визначення парціальної ентальпії розчинення і інших величин в досліджуваних системах. Така послідовність розрахунків є запропонованим методом визначення термодинамічних параметрів розчинення. Визначені значення і температурні залежності ентальпії розчинення малорозчинного компонента для таких систем як Cd-Na, Cd-Tl, Te-Ga, Te-As, Te-Cu, Zn-Sn, а також коефіцієнти активності $\gamma_{B}^{\alpha}$ і активності $a_{B}^{\alpha}$ другого компонента в насиченій $\alpha$-фазі в цих системах. Аналіз отриманих результатів по ентальпії розчинення показує, що спостерігається зміна парціальної ентальпії зі зміною температури розчинення. При зниженні температури від точки плавлення чистого основного компонента до температури евтектики спостерігається збільшення парціальних ентальпій розчинення другого компонента для досліджених систем. Система Те-Gа характеризується від'ємним значенням $\Delta \bar{H}_{B}^{\alpha}$. Негативне значення $\Delta \bar{H}_{B}^{\alpha}$ вказує на екзотермічний процес розчинення галію в телурі, на відміну від інших систем, в яких розчинення другого компонента відбувається з поглинанням тепла. Аналізуючи значення ентальпії розчинення в досліджуваних системах, необхідно відзначити, що низьким значенням $\Delta \bar{H}_{B}^{\alpha}<2000$ Дж/моль характеризуються системи Cd-Na i Te-Ga. Для систем Te-Cu, Te-As, Te-Tl, Zn-Sn> 15000 Дж/моль. Системи зі значенням $\Delta \bar{H}_{B}^{\alpha}>15000$ Дж/моль виявляють тенденцію до виникнення в них ретроградної розчинності. Виявлено відмінність у значеннях $\gamma_{B}^{\alpha}$ більш, ніж на два порядки для різних систем.

КЛЮЧОВІ СЛОВА: подвійні металеві системи, $\alpha$-фаза, ентальпія розчинення, коефіцієнт активності, активність, ретроградна розчинність, діаграма стану

Вопросы образования твердых растворов занимают важное место в термодинамике твердого состояния. Как в природе, так и в технике постоянно приходится иметь дело с растворами, а не с чистыми компонентами. Чистые вещества представляют собой лишь предельное состояние, которое никогда в действительности не достигается. Получаемые в современной технике методами вакуумной дистилляции и зонной плавкой высокочистые металлы все же содержат ничтожные количества $\left(10^{-6} \%\right)$ примесей и по существу являются растворами.

Задачей физико-химического анализа является установление зависимости между составом и свойством сложных систем. Для металловедения и металлургии, в том числе и расчета эффективности получения высокочистых металлов и сплавов, представляет особый интерес в качестве основного свойства выбрать температуры равновесия между жидкой и твердой фазами, а также температуры фазовых равновесий в твердом состоянии. Основные типы фазовых равновесий в виде диаграмм состояния (ДС) представлены в работе [1].

При образовании твердых растворов и равновесии между фазами различного состава одним из основных термодинамических параметров является парциальная энтальпия растворения $\Delta \bar{H}_{B}^{\alpha}$ компонента В в твердой $\alpha$-фазе. Другими важными термодинамическими величинами являются коэффициенты активности $\gamma_{B}^{\alpha}$ и сами активности $a_{B}^{\alpha}$ растворяемого элемента. Представляет практический интерес исследования этих термодинамических величин при растворении малорастворимых компонентов в основе.

В данной работе предлагается алгоритм определения значений выше указанных параметров в бинарных металлических системах с низкой растворимостью компонентов в твердом состоянии. Расчеты выполнены для диаграмм равновесия, в которых для упрощения с достаточным приближением считается, что из жидкости выделяются в виде отдельных фаз чистые компоненты.

\section{ПОСТАНОВКА ЗАДАЧИ}

Поскольку абсолютной нерастворимости компонентов друг в друге не существует, на ДС должна быть линия ограниченной растворимости $\alpha$-фазы как функция температуры и состава. Отсутствие таких линий связано с тем, что в некоторых двойных системах области твердых растворов $\alpha$-фазы настолько узкие $(<1$ ат.\%), что точное экспериментальное определение их является затруднительным. К такому типау ДС относятся многие системы, такие как $\mathrm{Cd}-\mathrm{Na}, \mathrm{Cd}-\mathrm{Tl}, \mathrm{Te}-\mathrm{As}, \mathrm{Te}-\mathrm{Cu}, \mathrm{Te}-\mathrm{Ga}, \mathrm{Zn}-\mathrm{Sn}$ и др.

Системы с такой низкой растворимостью можно отнести к системам с "вырожденной” эвтектикой, изображение области первичных твердых растворов которых в обычных масштабах на ДС не представляется возможным (воображаемые линии солидуса совпадают с осью ординат чистых компонентов). Визуальное представление таких воображаемых областей предельного растворения возможно при переходе к логарифмическим 
координатам изображения.

Ранее авторами данной работы были подробно исследованы как выше перечисленные, так и другие системы такого типа на предмет определения в них предельных $k_{\text {olimB }}$ и равновесных $k_{0 B}$ коэффициентов распределения компонента В при кристаллизации из расплава [2-4]. Значения этих величин были использованы для расчета предельной растворимости вторых компонентов $X_{B \text { (насыщ) }}^{\alpha}$ и построения линий солидуса диаграмм состояния [5-7]. Полученные в перечисленных выше работах данные использованы в данной работе для определения парциальной энтальпии растворения $\Delta \bar{H}_{B}^{\alpha}$ и других величин в исследуемых системах. Такая последовательность расчетов является предлагаемым алгоритмом определения термодинамических параметров растворения.

Справочные данные по значению термодинамических величин растворения весьма ограничены, а для металлических систем с низкой растворимостью компонентов и вовсе отсутствуют.

Целью работы является определение по предложенному алгоритму значений таких термодинамических величин, как парциальная энтальпия растворения $\Delta \bar{H}_{B}^{\alpha}$, коэффициент активности $\gamma_{B}^{\alpha}$ и активность $a_{B}^{\alpha}$ второго компонента в бинарных металлических системах с низкой растворимостью в твердой $\alpha$-фазе.

\section{МЕТОДИКА РАСЧЕТОВ, РЕЗУЛЬТАТЫ И ИХ ОБСУЖДЕНИЕ}

Для определения $\Delta \bar{H}_{B}^{\alpha}$ необходимо иметь такое соотношение, которое бы связывало эту величину с известными справочными термодинамическими величинами, такими как энтальпии и энтропии плавления основного и второго компонента, а также концентрациями насыщения компонента В в твердой $\alpha$-фазе и жидкой фазе в зависимости от температуры, что представляется линиями солидуса и ликвидуса на диаграммах состояния. В работе [8] получено такое выражение:

$$
\ln \frac{X_{\mathrm{B}(\text { насыш })}^{\alpha}}{\mathrm{X}_{\mathrm{B}}^{\text {ӝ }}}=\frac{\Delta H_{\text {плВ }}-\Delta \bar{H}_{B}^{\alpha}}{R T}-\frac{\Delta S_{\text {плВ }}}{\mathrm{R}},
$$

где $X_{\mathrm{B}(\text { насыщ) }}^{\alpha}$ - максимальная концентрация растворенного компонента В в $\alpha$-фазе при температуре $T ; X_{\mathrm{B}}^{\text {ж}}$ концентрация растворенного компонента В в жидкой фазе; $\Delta H_{\text {плв }}$ - изменение энтальпии при плавлении компонента В; $\Delta \bar{H}_{B}^{\alpha}$ - парциальная энтальпия растворения компонента В в $\alpha$-фазе; $R$ - универсальная газовая постоянная; $\Delta S_{\text {плВ }}$ - изменение энтропии при плавлении компонента В.

Вывод искомого выражения выполнен в предположении, что $\alpha$-фаза ведет себя как регулярный раствор, а жидкая фаза, находящаяся в равновесии с $\alpha$-фазой выше эвтектической температуры, ведет себя как идеальный раствор. Отношение $X_{\mathrm{B}(\text { насыщ) }}^{\alpha} / X_{B}^{\text {ж}}$ известно как коэффициент распределения, его часто обозначают символом $k_{0 B}$.

Из зависимости (1) получаем аналитическое выражение (2) для определения парциальной энтальпии растворения компонента В в $\alpha$-фазе, которое использовалось для расчетов в данной работе.

$$
\Delta \overline{\mathrm{H}}_{\mathrm{B}}^{\alpha}=-R T \ln \frac{X_{\mathrm{B}(\text { насыщ) }}^{\alpha}}{X_{B}^{ж}}+\Delta H_{\text {плВ }}-T \Delta S_{\text {плв }}
$$

Для расчетов значений $\Delta \bar{H}_{B}^{\alpha}$ отношения $X_{\text {В(насыш) }}^{\alpha} / X_{B}^{\text {ж}}$ для каждой системы брали из работы [7]. Справочные данные для расчетов по энтальпии и энтропии плавления растворенного компонента взяты из работы [9].

Для исследованных систем Cd-Na, Cd-Tl, Te-As, Te-Cu, Te-Ga, Zn-Sn температурная зависимость значений $\Delta \bar{H}_{B}^{\alpha}$, рассчитанная по формуле (2), представлена на рис. 1.

Зная величину $\Delta \bar{H}_{B}^{\alpha}$, можно из выражения (3), которое также было получено в работе [8] при выводе зависимости (1), определить коэффициент активности $\gamma_{B}^{\alpha}$.

$$
R T \ln \gamma_{B}^{\alpha}=\Delta \bar{H}_{B}^{\alpha}
$$


где, $\gamma_{B}^{\alpha}$ - коэффициент активности компонента В в насыщенной $\alpha$-фазе при температуре Т; $\Delta \bar{H}_{B}^{\alpha}, R$ и $T$ и - те же величины, что и в (1).
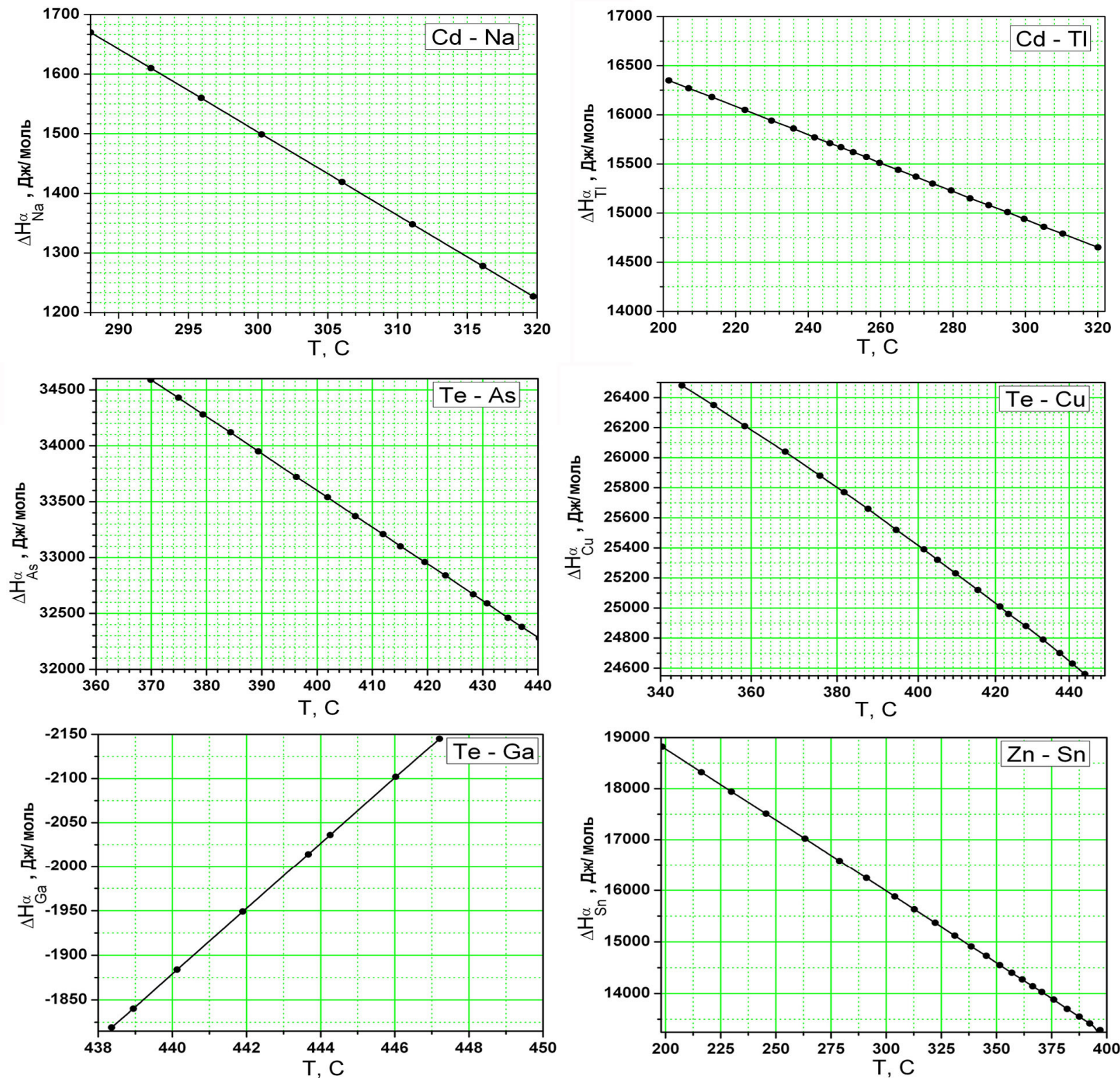

Рис. 1. Зависимость энтальпии растворения примеси в металле от температуры для систем: $\mathrm{Cd}-\mathrm{Na}, \mathrm{Cd}-\mathrm{Tl}, \mathrm{Te}-\mathrm{As}, \mathrm{Te}-\mathrm{Cu}, \mathrm{Te}-$

$\mathrm{Ga}, \mathrm{Zn}-\mathrm{Sn}$.

На рис. 2 представлены графические значения коэффициентов активности $\gamma$ как функции обратной температуры для исследованных систем.

Анализ полученных результатов по энтальпии растворения показывает, что наблюдается изменение парциальной энтальпии с изменением температуры растворения. При снижении температуры от точки плавления чистого основного компонента до температуры эвтектики наблюдается увеличение парциальных энтальпий растворения второго компонента. Из общей закономерности положительных значений парциальных энтальпий растворения для исследованных систем отличительную особенность проявляет система Te-Ga, которая характеризуется отрицательным значением $\Delta \bar{H}_{B}^{\alpha}$. Отрицательное значение $\Delta \bar{H}_{B}^{\alpha}$ указывает на экзотермический процесс растворения галлия в теллуре, в отличие от других систем, в которых растворение второго компонента происходит с поглощением тепла. 

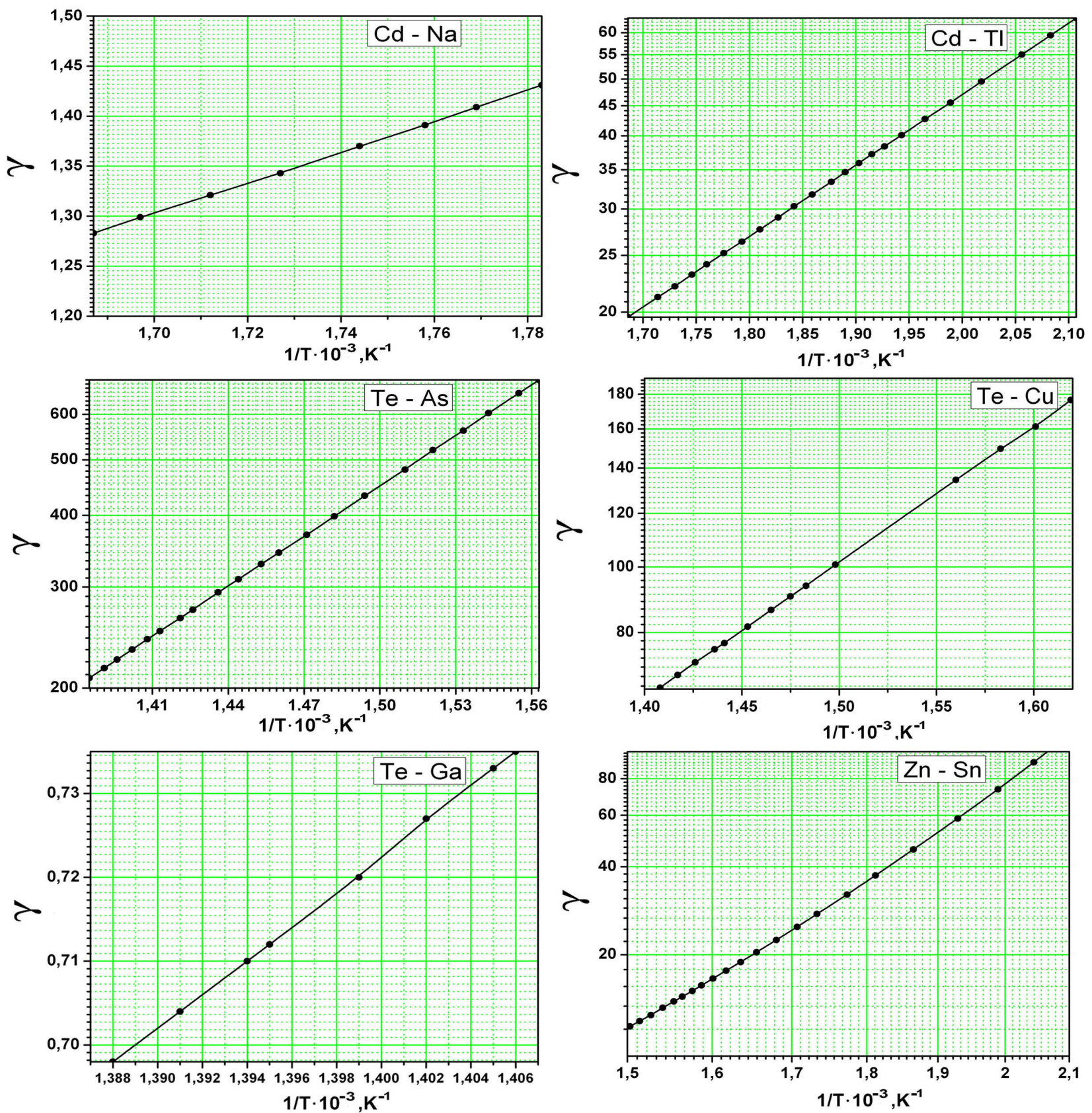

Рис. 2. Значения коэффициента активности примеси в твердой $\alpha$-фазе в зависимости от обратной температуры для систем: Cd-Na, Cd-Tl, Te-As, Te-Cu, Te-Ga, Zn-Sn.

Анализируя значения энтальпии растворения в исследуемых системах, необходимо отметить, что низким значением $\Delta \bar{H}_{B}^{\alpha}<2000$ Дж/моль характеризуются системы Cd-Na и Te-Ga. Для систем Te-Cu, Te-As, Te-Tl, $\mathrm{Zn}-\mathrm{Sn} \Delta \bar{H}_{B}^{\alpha}>15000$ Дж/моль. Построенные для этих систем линии солидуса [7] выявляют тенденцию к возникновению в них ретроградной растворимости, что можно связать с высокими значениями $\Delta \bar{H}_{B}^{\alpha}$ в этих системах.

В работах $[8,10]$ приводятся данные о ретроградной растворимости малорастворимых примесей в Ge и $\mathrm{Si}$. Авторы этих работ связывают явление отрицательной (ретроградной) растворимости с относительной парциальной энтальпией растворенного вещества в твердом растворителе. Как показал расчет, для систем с относительно низким значением $\Delta \bar{H}_{B}^{\alpha}$ не обнаруживается тенденции к ретроградной растворимости, но с увеличени- 
ем значения парциальной энтальпии тенденция к ретроградной растворимости растет.

Анализ зависимостей коэффициентов активности $\gamma_{B}^{\alpha}$ от обратных температур (рис. 2 показывает, что значения $\gamma_{B}^{\alpha}$ для различных систем существенно (на два порядка) разнятся.

Зная коэффициенты активности второго компонента в $\alpha$-фазе можно определить и саму активность $a_{B}^{\alpha}$ компонента В в $\alpha$-фазе в зависимости от концентрации насыщения. Активность определяли, используя следующее выражение

$$
a_{B}^{\alpha}=\gamma_{B}^{\alpha} X_{\mathrm{B} \text { (насыщ) }}^{\alpha}
$$

В таблице приведены расчетные значения полученных термодинамических величин второго компонента в системах Cd-Na, Cd-Tl, Te-As, Te-Cu, Te-Ga, Zn-Sn для трех значений температур и соответствующих им концентраций.

Таблица

Термодинамические величины и их значения для выбранных температур и концентраций

\begin{tabular}{|c|c|c|c|c|c|}
\hline Система & $\mathrm{T}, \mathrm{C}$ & Концентрация, ат.\% & Н, Дж/моль & $\gamma$ & Активность, \% \\
\hline \multirow{3}{*}{$\mathrm{Cd}-\mathrm{Na}$} & 316,12 & 0,626 & 1278 & 1,299 & 0,813 \\
\hline & 306,02 & 1,561 & 1419 & 1,343 & 2,097 \\
\hline & 288 & 2,624 & 1670 & 1,431 & 3,756 \\
\hline \multirow{3}{*}{$\mathrm{Cd}-\mathrm{Tl}$} & 295,09 & 0,252 & 15010 & 24,127 & 6,074 \\
\hline & 256,08 & 0,902 & 15570 & 34,66 & 31,257 \\
\hline & 222,55 & 1,404 & 16050 & 49,51 & 69,526 \\
\hline \multirow{3}{*}{$\mathrm{Te}-\mathrm{As}$} & 443,35 & 0,013 & 32180 & 224,148 & 2,924 \\
\hline & 440,21 & 0,019 & 32280 & 233,462 & 4,427 \\
\hline & 406,94 & 0,059 & 33370 & 369,807 & 21,784 \\
\hline \multirow{3}{*}{$\mathrm{Te}-\mathrm{Ga}$} & 446,03 & 0,369 & -2102 & 0,703 & 0,259 \\
\hline & 443,67 & 0,932 & -2014 & 0,713 & 0,664 \\
\hline & 438,95 & 1,548 & -1840 & 0,732 & 1,134 \\
\hline \multirow{3}{*}{$\mathrm{Zn}-\mathrm{Sn}$} & 403.12 & 0,241 & 11830 & 8,232 & 1,984 \\
\hline & 356,93 & 1,450 & 14400 & 15,708 & 22,777 \\
\hline & 229,89 & 1,095 & 17940 & 73,556 & 80,544 \\
\hline \multirow{3}{*}{$\mathrm{Te}-\mathrm{Cu}$} & 440,88 & 0,145 & 24660 & 64,191 & 9,311 \\
\hline & 415,37 & 0,464 & 25150 & 81,603 & 37,898 \\
\hline & 351,60 & 0,695 & 26380 & 161,226 & 112,035 \\
\hline
\end{tabular}

Температуры выбраны вблизи температуры плавления чистого компонента и вблизи температуры эвтектики с одним промежуточным значением. Отношение активностей в крайних точках значительно ( в 10 раз) отличается от системы к системе. Например, для системы Cd-Na оно равно 4, а для Zn-Sn - 40,5.

\section{ВЫВОДЫ}

Предложен расчетный метод определения значений таких термодинамических величин, как парциальная энтальпии растворения $\Delta \bar{H}_{B}^{\alpha}$, коэффициент активности $\gamma_{B}^{\alpha}$ и активность $a_{B}^{\alpha}$ второго компонента В в двойных металлических системах с низкой растворимостью В в твердой $\alpha$-фазе.

Определены значения и температурные зависимости энтальпии растворения малорастворимого компонента для систем Cd-Na, Cd-Tl, Te-As, Te-Cu, Te-Ga, Zn-Sn. Выявлено, что системы Te-Cu, Te-As, Te-Tl, Zn-Sn co значением $\Delta \bar{H}_{B}^{\alpha}>15000$ Дж/моль проявляют тенденцию к возникновению в них ретроградной растворимости, что можно связать с высокими значениями $\Delta \bar{H}_{B}^{\alpha}$ в этих системах.

Построены графические зависимости коэффициентов активности $\gamma_{B}^{\alpha}$ от температуры. Значения коэффициентов активности в различных двойных системах отличаются более чем на два порядка.

Определены активности $a_{B}^{\alpha}$ малорастворимых компонентов в насыщенной твердой $\alpha$-фазе в этих системах в зависимости от температуры и концентрации. Отношение активностей вблизи температуры плавления чистого компонента и вблизи температуры эвтектики значительно отличается в разных системах. Так, для системы Cd-Na оно равно 4, а для системы $\mathrm{Zn}-\mathrm{Sn}-40,5$. 


\section{СПИСОК ЛИТЕРАТУРЫ}

1. Lyakishev N.P. Phase diagrams of binary metal system. - Vol.1. - M.: Mashinostroyeniye, 1996. - 992 p.; Vol.2. - M.: Mashinostroyenoye, 1997. - 1024 p. (in Russian)

2. Kovtun G.P., Scherban' A.P., Datsenko O.A. Determination of the limiting impurity distribution coefficients $\mathrm{k}_{0} \operatorname{limB}_{\mathrm{B}}$ in cadmium and zinc // Voprosy atomnoy nauki i tekhniki. Ser.: Vacuum, pure materials, superconductors. - 2002. - No. 1(12). P. 151-155. (in Russian)

3. Kovtun G.P., Scherban' A.P., Datsenko O.A. Calculation method for determining the limiting impurity distribution coefficients $\mathrm{k}_{0 \mathrm{limB}}$ at the directional crystallization of metals // Voprosy atomnoy nauki i tekhniki. Ser.: Vacuum, pure materials, superconductors. - 2003. - No. 5(13). - P. 3-6. (in Russian)

4. Scherban' A.P. The limiting distribution coefficients of low-soluble impurities in metals // Journal of V.N. Karazin Kharkiv National University. Series Nuclei, particles, fields. - 2011. - No. 946. - Iss.1(49) - P. 75-80. (in Russian)

5. Kovtun G.P., Scherban' A.P. Estimation of the solubility limit of low-soluble components under eutectic transformations in the binary metal systems // Problem of Atomic Science and Engineering. - 2014. - No. 1(89). - P. 37-40.

6. Scherban' A.P., Datsenko O.A. Analysis of phase diagrams of binary metal systems in the field at low concentration of components // Journal of V.N. Karazin Kharkiv National University. Series Nuclei, particles, fields. - 2013. - No. 1069. - Iss. 4(60). - P. 95-99. (in Russian)

7. Shcherban A.P., Datsenko O.A., Kovtun G.P. Construction of Solidus Lines of Binary Metal Systems Having a Low Solubility of Components in the Solid Phase // Open Journal of Metal. - 2014. - Vol. 4. - P. 65-71.

8. Svellin R.A. Solid phase thermodynamics. - Metallurgiya, 1968. - 316 p.

9. Barthel J., Buhrig E., Hein K., Kuchař L. Cristallisation aus Schmelzen // Reference manual: Translation from German. - M.: Metallurgy, 1987. -320 p. (in Russian).

10. Kröger F.A. Chemistry of imperfect crystals. - Translation under the editorship of Poltorak O.M., 1969. - 654 p. 